\title{
Algorithm Clinical Reasoning for Management of Invasive Carcinoma of Breast
}

\author{
Francisca Farrar* \\ Department of Nursing, Austin Peay State University, USA
}

*Corresponding author: Francisca Farrar, Department of Nursing, Austin Peay State University, Clarksville, USA.

Received Date: May 20, 2019

Published Date: May 28, 2019

\begin{abstract}
A 65-year-old female had an abnormal mammogram during her annual screening showing an $8 \mathrm{~mm}$ mass in the right breast. She is postmenopausal with the onset of menarche at 11 years and menopause at 50 years. She is a widow with two children with the first live birth at 30 year and second girl at 36. This 65-year female was on hormone replacement for 10 years due to migraine headaches. She has not had a hysterectomy. This case report uses this medical history and pathology report to identify variables for a clinical reasoning algorithm to develop a survival plan of care. A holistic plan is developed for her surgery, adjunctive therapy, diet, and integrative medicine to treat mind, body, and spirit. Preventive care is also discussed.
\end{abstract}

Keywords: Invasive breast carcinoma mammogram; Pathology report genetic testing; Integrative medicine; Mediterranean diet

\section{Introduction}

Breast cancer screening with an annual mammogram and selfexamination for a lump in the breast can identify breast cancer at an early age [1]. In the United States breast cancer is the second leading cause of death in women [2]. These preventive measures can diagnose breast cancer early and increase survival rate. Most cancers start in cells that line milk glands with milk dividing with genetic mutations turning the healthy normal cells into cancer cells that can form a mass and invade other vital organs in the body. Ductal and lobular are two main types of breast cancer. Lobular carcinoma is found in the milk producing glands while ductal carcinoma is found in the passageways that carry milk from the lobules to the nipple. Breast cancer is diagnosed as noninvasive called carcinoma in situ and invasive carcinoma that can spread to the lymph nodes and invade vital organs such as the lung and liver. The most common breast carcinoma is invasive ductal carcinoma at $75 \%$ while invasive lobular carcinoma accounts for 10 to $15 \%$ of carcinoma $[3,4]$. The following case report about a female diagnosed with invasive ductal carcinoma. The case report and carcinoma management demonstrate clinical reasoning and applying an algorithm of variables for treatment of this carcinoma.

\section{Case Report}

A 65-year-old female had an abnormal mammogram during her annual screening showing an $8 \mathrm{~mm}$ mass in the right breast.
She is post-menopausal with the onset of menarche at 11 years and menopause at 50 years. She is a widow with two children with the first live birth at 30 year and second girl at 36. This 65-year female was on hormone replacement for 10 years due to migraine headaches. She has not had a hysterectomy.

\section{Diagnostic Evaluation of the Carcinoma}

A real-time sonography was done to evaluate this tumor. The right breast ultrasound confirmed an $8 \mathrm{~mm}$ tumor. An ultrasound guided core biopsy was performed. The pathology report confirmed invasive carcinoma with prominent tubule formation, moderate nuclear atypica, and 6 mitoses per 10 . The tumor measured $0.5 \mathrm{~cm}$ with no evidence of lymph vascular invasion. The female was referred to a cancer center for treatment of her invasive carcinoma in her right breast.

\section{Pathology Report}

The pathology report proved there was an $8 \mathrm{~mm}$ malignancy within the right breast at the 10 o'clock position. There were no abnormal lymph nodes identified within the right axilla. Pathology demonstrated stage 1 and grade 2 invasive ductal carcinoma. The estrogen receptor was positive in approximately $95 \%$ of tumor cell nuclei, progesterone receptor was positive in $95 \%$ of tumor, and HER 2 is negative. Ki-67 was $20 \%$. The stage of carcinoma reflects 
the invasive status and biology of the tumor such as hormone and HER 2 status. Normal breast tissue cells contain receptors for hormones estrogen and progesterone. When cancer occurs, these receptors can be retained. The cancer cells can be fed by these hormones. Breast cells can also be activated by human epidermal growth factor receptor called HER 2 protein. The proliferation rate (Ki67) provides information about how fast the cancer tumors divide. Breast cancer is graded 1 to 3 to determine aggressive growth of carcinoma [5].

\section{Clinical Reasoning Algorithm Using Pathology Variables to Develop a Survival Plan of Care}

\section{Surgery treatment is required for removal of the invasive ductal carcinoma}

Based on the Stage 1, grade2, HER 2 negative, and KI 167 pathological report plus early cancer detection with a small tumor provided this 65-year female the option of selecting a mastectomy or lumpectomy followed by radiation. This 65-year-old female requested genetic counseling and testing prior to making this decision. Genetic testing was negative for BRCA1 and CRCAs gene mutation [6]. Sentinel node biopsy was performed during surgery with the four sentinel nodes identified by radioactive dye injected prior to surgery were removed. During surgery a frozen section of margins of the lumpectomy and dissected nodes were evaluated. The margins were clear, and all sentinel lymph nodes were negative for cancer cells. The final support supported clear margins and negative sentinel nodes for spread of cancer. Radiation is required after the lumpectomy [9]. External hypo fractional whole breast radiation was ordered for three weeks at five times a week. A nutritional consult educated her about the need for protein and hydration to prepare her skin for radiation. The Mediterranean diet was recommended $[7,8]$.

\section{Adjuvant therapy is needed}

The goal of adjuvant therapy is to destroy any remaining microscopic cancer cells. Hormonal therapy also called endocrine therapy blocks hormone receptors. These medications prevent estrogen from binding to estrogen receptors. Hormonal therapy improves survival rates and decreases the chance for cancer to return in the breast or vital organs [3,4]. Tamoxifen and Aromatase inhibitors are the two common drugs used for hormonal therapy. Since this 65-year female is post-menopausal and medical history of no hysterectomy the aromatase inhibitor was chosen. Education to this 65-year female is common side effects are joint pain, joint stiffness, hot flashes, and vaginal dryness. This medication should be taken at the same time every day without missing a dose for 5 years. She should also wash her hands after taking the pill.

\section{Integrative therapy is needed to maintain healthy mind, body, and spirit}

This female patient grieved over the threat of death and losing her right breast. She reported shock, denial, anger and depression during the treatment. The plan of care included a holistic approach that included help to deal with her voiced grief, depression, anxiety, and fear. These 65 females were referred to an integrative medicine department for resources to develop a wellness program. MindBody therapies suggested were guided mindfulness mediation, yoga, pet therapy, relaxation, spirituality, massage, and acupressure $[10,11]$. She chose all but acupressure.

\section{Prevention follow-up care}

This 65-year-old patient will need to have 6-month mammograms. She will need to develop a healthy lifestyle by following the Mediterranean diet, develop an exercise program, and using integrative therapy [4].

\section{Acknowledgement}

None.

\section{Conflict of interest}

I have no conflict of interest.

\section{References}

1. Centers for Disease and Prevention. What is Breast Cancer Screening.

2. (2018) US Cancer Statistics Working Group. US Cancer Statistics Data Visualizations Tool, based on November 2017 submission data (19992015): US Department of Health and Human Services, Centers for Disease Control and Prevention and National Cancer Institute, USA.

3. Center for Disease Control and Prevention. Basic Information about Breast Cancer.

4. Center for Disease Control and Prevention. What is Breast Cancer?

5. National Cancer Institute Pathologic Evaluation of Breast Tissue.

6. National Cancer Institute. The Genetics of Cancer.

7. Mayo Clinic. Patient Education. Mediterranean Diet.

8. American Institute for Cancer Research. Nutrition of the Cancer Patient. E21-NP, Washington, DC, USA.

9. (2016) National Institute of Health National Cancer Institute. Radiation Therapy and You: Support for People with cancer. NIH Publication pp: 7117-7157.

10. (2005) National Cancer Institute. National Center for Complementary and Alternative Medicine. Eating Hints: Before during, and after Cancer Treatment. NIH Publication pp: 04-55.

11. Mayo Clinic. Patient Education. Exploring Complementary and Integrative Therapies. 